\title{
SOLUTIONS WITH COMPACT SUPPORT OF THE POROUS MEDIUM EQUATION IN ARBITRARY DIMENSIONS
}

\author{
MICHIAKI WATANABE
}

(Communicated by John B. Conway)

\begin{abstract}
Compactness of the support is discussed of a solution $u$ to the Cauchy problem for the porous medium equation $u_{t}=\Delta \phi(u), t>0$, in $R^{N}$ of arbitrary dimension $N \geq 1$, where $\phi$ is a nondecreasing function on $R^{1}$. It is shown that if $u(0, x)=0$ for $|x| \geq R, R>0$, then for all $t \geq 0$

$$
u(t, x)=0 \text { a.e. }|x| \geq R+C t^{1 / 2}
$$

with a constant $C$ depending on $\phi$ and $u(0, \cdot)$.

The result is well known when $N=1$, but the study for $N>1$ has somehow been neglected.
\end{abstract}

1. Statement of the result. Consider the Cauchy problem for the porous medium equation in $R^{N}$ for $N \geq 1$ :

$$
\begin{gathered}
u_{t}=\Delta \phi(u), \quad t>0, x \in R^{N} ; \\
u(0, x)=u_{0}(x), \quad x \in R^{N} .
\end{gathered}
$$

In the case of $\phi(r)=r$, the solution is given by

$$
(4 \pi t)^{-N / 2} \int_{R^{N}} e^{-|x-y|^{2} / 4 t} u_{0}(y) d y
$$

and is shown to spread out in $R^{N}$ for any $t>0$ unless $u_{0}(x)=0$ for a.a. $x \in R^{N}$. But the situation is different if $\phi(r)=|r|^{m-1} r, m>1$, for example. The purpose of this paper is to show under more general assumptions on $\phi$ that if

$$
u_{0} \in L^{\infty}\left(R^{N}\right) ; \quad u_{0}(x)=0 \text { for }|x| \geq R, R>0,
$$

then the solution (in a generalized sense) $u(t, x)$ of (1) and (2) stays compactly supported in $R^{N}$ forever: For all $t \geq 0$

$$
u(t, x)=0 \text { a.e. }|x| \geq R+C t^{1 / 2}
$$

with a constant $C>0$ depending on $\phi$ and $\left\|u_{0}\right\|_{\infty}$.

This kind of phenomenon is well known when $N=1$ : Even the lateral boundary, called the interface, of the support of $u(t, x)$ in $R^{N}$ has been discussed extensively together with its behavior (see e.g. $[4,5]$ ). However, the study in the case of $N>1$ has somehow been neglected. Within the Cauchy problem (1)-(2) little is known about compact support of the solution (cf. Diaz Diaz [3] and Schatzman [6]).

Received by the editors March 13, 1987. This paper was presented at Sapporo Symposium on Partial Differential Equations held in Hokkaido University, Japan, July 27, 1987.

1980 Mathematics Subject Classification (1985 Revision). Primary 35K15, 35K55.

Key words and phrases. Cauchy problem for porous medium equation, solution with compact support. 
In this paper we shall deal with solutions of $(1)$ in $D^{\prime}\left((0, \infty) \times R^{N}\right)$ (in the sense of distributions on $\left.(0, \infty) \times R^{N}\right)$ under the following conditions on $\phi$ :

$\phi$ is a nondecreasing continuous function on $R^{1}$ with $\phi(0)=0$;

the range of $\phi$ coincides with $R^{1}$, and for each $r \in R^{1}$ the integral $\int_{0}^{r} 1 / \phi^{-1}(s) d s$ exists.

Based on the work [1], Brézis and Crandall [2] established that, for each $u_{0} \in$ $L^{1}\left(R^{N}\right) \cap L^{\infty}\left(R^{N}\right)$, there is a unique solution $u$ of $(1)-(2)$ in $D^{\prime}\left((0, \infty) \times R^{N}\right)$ such that

$$
u \in C\left([0, \infty) ; L^{1}\left(R^{N}\right)\right) \cap L^{\infty}\left((0, \infty) \times R^{N}\right) .
$$

It is suggested in the paper [2, (1.20)] that for each $T>0$,

$$
u_{0} \in L^{1}\left(R^{N}\right) \cap L^{\infty}\left(R^{N}\right) ; \quad f \in L^{1}\left(0, T ; L^{1}\left(R^{N}\right) \cap L^{\infty}\left(R^{N}\right)\right),
$$

there exists a unique solution $u$ in $D^{\prime}\left((0, T) \times R^{N}\right)$ of

$$
u_{t}=\Delta \phi(u)+f(t, x), \quad 0<t<T, x \in R^{N},
$$

and (2) such that

$$
u \in C\left([0, T] ; L^{1}\left(R^{N}\right)\right) \cap L^{\infty}\left((0, T) \times R^{N}\right) .
$$

The main result is formulated as follows:

THEOREM. Under the conditions (3)-(5), the solution $u$ in $D^{\prime}\left((0, \infty) \times R^{N}\right)$ of (1)-(2) with (6) satisfies, for all $t \geq 0$,

$$
u(t, x)=0 \quad \text { a.e. }|x| \geq R+2 C_{0} t^{1 / 2}
$$

with

$$
C_{0}=\left\{\int_{0}^{\phi\left(\left\|u_{0}\right\|_{\infty}\right)} \frac{1}{\phi^{-1}(s)} d s\right\}^{1 / 2} .
$$

The theorem coincides with a result for $N=1$ of Knerr [4, Theorem 8.4] and is similar to a result of $\operatorname{Diaz} \operatorname{Diaz}$ [3, Theorem I] for an initial-boundary value problem.

2. Proof of the main result. The difficulty in this paper is that no exact solution of (1) can be found, for $N$ is arbitrary and the explicit form of $\phi(r)$ is not necessarily given. So the main task is to show that the function $v=v(t, x)$, determined by

$$
\int_{0}^{\phi(v)} \frac{1}{\phi^{-1}(s)} d s=\max \left(0, a-\frac{|x|^{2}}{4(t+b)}\right),
$$

where $a$ and $b$ are positive constants, is compactly supported and a solution in $D^{\prime}\left((0, T) \times R^{N}\right)$ of $(1)^{\prime}$ with a nonnegative and "small" function $f(t, x)$. If it is done, the proof is carried out as usual by using the comparison principle (Lemma 2 ) for solutions of $(1)^{\prime}-(2)$.

The function $y(r)$, defined under (4) and (5) by

$$
y(r)=\phi^{-1}\left(z^{-1}(r)\right) \quad \text { with } z(r)=\int_{0}^{r} \frac{1}{\phi^{-1}(s)} d s
$$


is a nondecreasing continuous function on $[0, \infty)$ with

$$
(d / d r) \phi(y(r))=y(r) \text { and } y(0)=0,
$$

and our function $v$ can be written as

$$
v(t, x)=y\left(\left(a-|x|^{2} / 4(t+b)\right)^{+}\right),
$$

where $r^{+}=\max (0, r)$ for $r \in R^{1}$. From this we see that $v$ is nonnegative valued, and satisfies

$$
v(t, x)=0 \text { for } t \geq 0 \text { and }|x|^{2} \geq 4 a(t+b)
$$

and, by Lebesgue's theorem, $(6)^{\prime}$ for each fixed $T>0$.

We shall show that $v$ is a solution in $D^{\prime}\left((0, T) \times R^{N}\right)$ of $(1)^{\prime}-(2)$ with $\left(u_{0} ; f\right)$ replaced by $\left(v_{0} ; g\right)$ :

$$
\left\{\begin{array}{l}
v_{0}(x)=y\left(\left(a-|x|^{2} / 4 b\right)^{+}\right) \\
g(t, x)=y\left(\left(a-|x|^{2} / 4(t+b)\right)^{+}\right) N / 2(t+b)
\end{array}\right.
$$

for which the condition (7) is valid.

LEMMA 1. For $t \geq 0$ and $x \in R^{N}, v$ satisfies

$$
\sum_{i=1}^{N} \frac{\partial}{\partial x_{i}} \int_{0}^{t} \frac{\partial}{\partial x_{i}} \phi(v) d r=[v(r, x)]_{0}^{t}-\int_{0}^{t} g(r, x) d r .
$$

PROOF. The proof is done by an elementary calculus. We have only to note (8) to see that

$$
\begin{aligned}
& \int_{0}^{t} \frac{\partial}{\partial x_{i}} \phi(v) d r=-\frac{2 x_{i}}{|x|^{2}}\left\{\left[(r+b) \phi\left(y\left(\left(a-\frac{|x|^{2}}{4(r+b)}\right)\right)^{+}\right)\right]_{0}^{t}\right. \\
& \left.-\int_{0}^{t} \phi\left(y\left(\left(a-\frac{|x|^{2}}{4(r+b)}\right)^{+}\right)\right) d r\right\}
\end{aligned}
$$

if $|x| \neq 0$, and equals 0 if $|x|=0$. Q.E.D.

Thus, multiplication by $\psi_{t}$ with $\psi \in C_{0}^{\infty}\left((0, T) \times R^{N}\right)$ of (10) and integration over $(0, T) \times R^{N}$ gives

$$
\int_{0}^{T} \int_{R^{N}}\left(v \psi_{t}+\phi(v) \Delta \psi+g(t, x) \psi\right) d x d t=0
$$

We are now in a position to examine the support in $R^{N}$ of the solution $u(t, x)$ in comparison with that of $v(t, x)$.

LEMMA 2. Take $T>0$ and let $\left(u_{0} ; f\right)$ and $\left(\hat{u}_{0} ; \hat{f}\right)$ satisfy the condition (7). Then the corresponding solutions $u$ and $\hat{u}$ in $D^{\prime}\left((0, T) \times R^{N}\right)$ of $(1)^{\prime}-(2)$ with $(6)^{\prime}$ satisfy

$$
\left\|(u(t, \cdot)-\hat{u}(t, \cdot))^{+}\right\|_{1} \leq\left\|\left(u_{0}-\hat{u}_{0}\right)^{+}\right\|_{1}+\int_{0}^{t}\left\|(f(r, \cdot)-\hat{f}(r, \cdot))^{+}\right\|_{1} d r
$$

for $0 \leq t \leq T$. 
PROOF. The lemma is a consequence of the property of the operator $\Delta \phi$ in $\left(L^{1}\left(R^{N}\right),\|\cdot\|_{1}\right)$ :

$$
\int_{R^{N}} \operatorname{sgn}^{+}(u-v) \cdot(\Delta \phi(u)-\Delta \phi(v)) d x \leq 0
$$

where

$$
\operatorname{sgn}^{+} r= \begin{cases}1 & (r>0) \\ 0 & (r \leq 0)\end{cases}
$$

The proof is standard and might be omitted (see $[3, \S 3 ; 7])$. Q.E.D.

PROOF OF THE THEOREM. Assume in addition that $u_{0}(x)$ is nonnegative for all $x \in R^{N}$ without loss of generality. Let $a$ and $b$ satisfy $a>R^{2} / 4 b$ and

$$
\left\|u_{0}\right\|_{\infty}=y\left(a-R^{2} / 4 b\right) \text {. }
$$

Then $y\left(\left(a-|x|^{2} / 4 b\right)^{+}\right)$is not smaller than $u_{0}(x)$ for a.a. $|x|<R$ and obviously for $|x| \geq R$, and hence

$$
0 \leq u_{0}(x) \leq v_{0}(x) \text { for a.a. } x \in R^{N} \text {. }
$$

Since $g(t, x)$ is nonnegative, we can apply Lemma 2 to the solutions $u(t, x)$ and $v(t, x)$ with data $\left(u_{0} ; 0\right)$ and $\left(v_{0} ; g\right)$ respectively to obtain

$$
0 \leq u(t, x) \leq v(t, x) \text { for a.a. } x \in R^{N}
$$

and for all $0 \leq t \leq T$ with each fixed $T>0$.

Recalling (9) with (11), we see that $u(t, x)$ vanishes for all $t \geq 0$ and a.a. $x \in R^{N}$ such that

$$
|x|^{2} \geq 4(t+b)\left\{R^{2} / 4 b+z\left(\phi\left(\left\|u_{0}\right\|_{\infty}\right)\right)\right\}, \quad b>0 .
$$

We have now only to find out the envelope. Choosing $b=(R / 2)\left\{t / z\left(\phi\left(\left\|u_{0}\right\|_{\infty}\right)\right)\right\}^{1 / 2}$ for $t>0$, we can conclude

$$
u(t, x)=0 \quad \text { a.e. }|x| \geq R+2\left\{z\left(\phi\left(\left\|u_{0}\right\|_{\infty}\right)\right) t\right\}^{1 / 2}
$$

for all $t \geq 0$. Q.E.D.

\section{REFERENCES}

1. Ph. Bénilan, H. Brézis, and M. G. Crandall, A semilinear elliptic equation in $L^{1}\left(R^{N}\right)$, Ann. Scuola Norm. Sup. Pisa Cl. Sci. (4) 2 (1975), 523-555.

2. H. Brézis and M. G. Crandall, Uniqueness of solutions of the initial value problem for $u_{t}-$ $\Delta \phi(u)=0$, J. Math. Pures Appl. 58 (1979), 153-163.

3. J. I. Diaz Diaz, Solutions with compact support for some degenerate parabolic problems, Nonlinear Anal., Theory, Methods and Appl. 3 (1979), 831847.

4. B. F. Knerr, The porous medium equation in one dimension, Trans. Amer. Math. Soc. 234 (1977), $381 \cdots 415$.

5. L. A. Peletier, The porous media equation, Applications of Nonlinear Analysis in the Physical Sciences (H. Amann, N. Bazley, and K. Kirchgässner, eds.), Pitman, 1981, pp. 229-241.

6. M. Schatzman, Stationary solutions and asymptotic behavior of a quasilinear degenerate parabolic equation, Indiana Univ. Math. J. 33 (1984), 129.

7. M. Watanabe, Trotter's product formula for semigroups generated by quasilinear elliptic operators, Proc. Amer. Math. Soc. 92 (1984), $509-514$.

FaCUlty Of General EduCation, Nigata University, IKarashi 2-8050, NiIgata, 950-21 JAPAN 